\title{
EFFICIENT HAPLOTYPE INFERENCE FROM PEDIGREES WITH MISSING DATA USING LINEAR SYSTEMS WITH DISJOINT-SET DATA STRUCTURES
}

\author{
Xin $\mathrm{Li}$ and Jing $\mathrm{Li}^{*}$ \\ Department of Electrical Engineering and Computer Science, Case Western Reserve University, \\ Cleveland, $\mathrm{OH}, 44106$ \\ *Email: jingli@case.edu
}

\begin{abstract}
We study the haplotype inference problem from pedigree data under the zero recombination assumption, which is well supported by real data for tightly linked markers (i.e., single nucleotide polymorphisms (SNPs)) over a relatively large chromosome segment. We solve the problem in a rigorous mathematical manner by formulating genotype constraints as a linear system of inheritance variables. We then utilize disjoint-set structures to encode connectivity information among individuals, to detect constraints from genotypes, and to check consistency of constraints. On a tree pedigree without missing data, our algorithm can output a general solution as well as the number of total specific solutions in a nearly linear time $O(m n \cdot \alpha(n))$, where $m$ is the number of loci, $n$ is the number of individuals and $\alpha$ is the inverse Ackermann function ${ }^{4}$, which is a further improvement over existing ones ${ }^{3}, 8,12,15$. We also extend the idea to looped pedigrees and pedigrees with missing data by considering existing (partial) constraints on inheritance variables. The algorithm has been implemented in $C++$ and will be incorporated into our PedPhase package ${ }^{8}$. Experimental results show that it can correctly identify all 0 -recombinant solutions with great efficiency. Comparisons with other two popular algorithms show that the proposed algorithm achieves 10 to $10^{5}$-fold improvements over a variety of parameter settings. The experimental study also provides empirical evidences on the complexity bounds suggested by theoretical analysis.
\end{abstract}

\section{INTRODUCTION}

Experimental data have shown that genetic variation is structured in haplotypes rather than isolated SNPs $^{14}$ and haplotypes may provide substantially increased power in detecting gene-disease association. However, the human genome is a diploid and, in practice, haplotype data are not collected directly, especially in large scale sequencing projects mainly due to cost considerations. Hence, efficient and accurate computational methods and computer programs for the inference of haplotypes from genotypes are highly needed.

Recent years have witnessed intensive research on haplotyping methods (see reviews ${ }^{2}, 5,6,10,17$ ), mainly driven by the HapMap project ${ }^{14}$. For family data, there exist two types of haplotyping methods, statistical methods and combinatorial (or rulebased) methods. And there is a tendency to merge these two types of approaches ${ }^{9,}{ }^{16}$. In general, the goal of statistical approaches ${ }^{1}$ is to find a haplotype assignment for each individual with the maximum likelihood or to output all consistent solutions with their corresponding probabilities. Recently, popu- lation haplotype frequencies have been taken into considerations $^{1}$ to account for correlations among tightly linked markers (known as linkage disequilibrium). A key step in most statistical approaches is to enumerate all possible inheritance patterns and to check the genotype consistency for each of them ${ }^{1}$. Due to the large degrees of freedom, this step usually leads to high time complexity (usually exponential hence computational intractable for large data sets). On the other hand, rule-based algorithms first partially infer haplotypes or inheritance vectors based on genotype constraints, and then search final solutions from the reduced space. Therefore, rule-based algorithms $3,8,9,12,15$ can potentially gain advantage over statistical methods in efficiency. The zero recombinant assumption states that recombination is nonexistent within a pedigree for a sufficiently large number of tightly linked markers. As a realistic assumption, it has been used in both statistical approaches as well as rule-based approaches. Furthermore, a solution to the problem with no recombinant can be served as a subroutine of a general procedure to solve the general haplotype inference problem. Therefore, investigation of efficient algorithms

\footnotetext{
${ }^{*}$ Corresponding author.
} 
to obtain all 0-recombinant solutions from a pedigree is of high interests.

For a given pedigree, the goal of the zero recombinant haplotype configuration (ZRHC) problem is to identify all possible haplotype assignments with no recombination. An important advance in the development of rule-based algorithms for haplotype inference in pedigrees in general and the ZRHC problem in particular is the introduction of variables to represent uncertainties. The problem can then be discussed and solved with mathematical rigor. $\mathrm{Li}$ and Jiang $^{8}$ first formulate the problem as a linear system on " $p s$ " (a binary indicator of parental source) variables and solve it using Gaussian elimination with a complexity of $O\left(m^{3} n^{3}\right)$, where $m$ is the number of markers and $n$ is the number of individuals. More recently, Xiao et al. ${ }^{15}$ formulate another linear system on " $h$ " (a binary indicator of inheritance relationship) variables, and lower the complexity to $O\left(m n^{2}+n^{3} \log ^{2} n \log \log n\right)$. For loop-free (tree) pedigrees, Xiao's method can produce a general solution in $O\left(m n^{2}+n^{3}\right)$ and a particular solution in $O\left(m n+n^{3}\right)$ time. Here, a particular solution means a specific assignment for each variable which satisfies the constraints, while a general solution is a description of all solutions in a general form where some variables are designated as free (meaning that they are allowed to take any value), and the remaining variables are represented by a linear combination of these free variables. For tree pedigrees, Chan et al. ${ }^{3}$ further reduce the complexity of finding a particular solution to a linear time $O(m n)$ by manipulating the constraints on a graph structure. Liu and Jiang ${ }^{12}$ also propose an algorithm to produce a particular solution in $O(m n)$ and a general solution in $O\left(m n^{2}\right)$ by further exploring features of their $h$ variable system on a tree pedigree. However, with missing data, it has been shown that ZRHC is NP-hard ${ }^{11}$. Therefore, it seems impossible to incorporate missing data into a pure linear constraint system without enumerations. Li and Jiang ${ }^{9}$ propose an integer linear programming algorithm for the minimum recombinant haplotype inference problem, and it can solve ZRHC with missing as a special case. But because it does not use zero recombinant constraints explicitly, it may need to enumerate almost all possible haplotype assignments.
In this paper, we propose an elegant and more efficient algorithm for detecting, recording and consistency checking of constraints on $h$ variables. Notice that it is not necessary to solve the $h$ variable system explicitly, as it was in Ref. 15. Instead, we encode constraints on $h$ variables using disjoint-set forests. By applying an adapted disjoint-set unionfind procedure, we can update the disjoint-set structures incrementally upon new constraints, and determine the consistency of the encoded linear system simultaneously. Based on the disjoint-set unionfind procedure, the proposed algorithm can produce a general solution in almost linear time $(O(m n \cdot \alpha(n))$ for a tree pedigree, where $\alpha$ is the inverse Ackermann function ${ }^{4}$, improved from the best known algorithm with $O\left(m n^{2}\right)$ time complexity ${ }^{12}$. We further extend the algorithm to looped pedigrees and pedigrees with missing data, by utilizing the constraints imposed from existing data. Experimental results show that the algorithm can output all solutions with zero recombinant and it is much more efficient than two popular existing algorithms because of the significant reduction of the enumeration space.

The rest of the paper is organized as follows. In section 2 , we introduce the linear system on $h$ variables together with some basic concepts and notations concerning the ZRHC problem. By representing constraints using a linear system, one can formally investigate different strategies to solve the problem in a rigorous manner. And different strategies of manipulating and integrating the constraints will result in different complexities. Our algorithm of detecting and processing constraints from pedigree data is presented in section 3 . In both sections, we assume input genotype data has no missing alleles and the ZRHC problem under this case is polynomially solvable. Our algorithm is almost optimal by achieving a nearly linear time complexity on tree pedigrees with complete data. In section 4 , we show how to extend the algorithm to cope with missing data and looped pedigrees by effectively reducing the search space before enumerations. The performance of our algorithm and comparisons with other two programs are examined in section 5. We discuss future directions and make concluding remarks in section 6 . 


\section{PRELIMINARIES}

A pedigree graph indicates the parent-child relationships among an extended family. Figures 1(a) and 2(a) present pedigrees in a conventional manner. The pedigree in Figure 1(a) has a mating loop, where an offspring (node 9 ) is produced by the mating of two relatives (node 6 and 7 ). A pedigree without mating loops is called a tree pedigree. A nuclear family only consists of both parents and their children. For any pair of homologous chromosomes from a diploid organism such as human, exactly one is from its father and the other one is from its mother, as illustrated in Figure 1(b). A physical position on a chromosome is called a locus and the status of a locus is called an allele, represented using an integer ID. We focus on SNP data in this study thus assume there are only two alternative alleles (i.e., biallelic data), which turns to be the hardest case for $\mathrm{ZRHC}^{8,15}$.

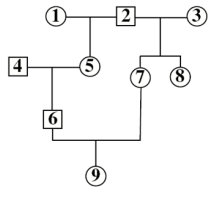

(a)

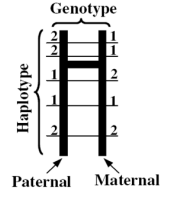

(b)

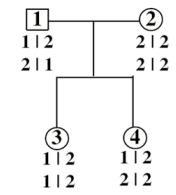

(c)
Fig. 1. (a) A pedigree graph. We use a circle to represent a female, a square to represent a male in a pedigree. (b) A haplotype is composed of all alleles on one chromosome segment. Each allele is an integer value representing the status of a marker at a chromosome locus. (c) A recombination event occurs when a child does not inherit a complete haplotype from its parent. Individual 3 has a paternal haplotype 11 which is not seen in his father. So there must be a crossover between two chromosomes of his father in meiosis, which results in a recombinant haplotype.

At each locus $i$, a child may inherit either of the paternal or maternal allele of a parent. We use binary variables to indicate parental source $(p s)$ of the two alleles in a child.

Definition 2.1. $p s$ variable $p_{i}^{x} \in\{0,1\}$ is defined for each locus $i$ of each individual $x . p_{i}^{x}=0$ if the smaller allele of locus $i$ is of paternal source, $p_{i}^{x}=1$ if it is of maternal source. We technically let $p_{i}^{x}=0$ if locus $i$ is homozygous (two alleles being the same).

Loosely speaking, a haplotype consists of all alleles on a chromosome. Recombination events or crossovers occur when a child inherits a shuffled version of its parent's two haplotypes (see Figure 1(c) for an example). However, for a sufficiently large segment of chromosome with $m$ SNPs, the likelihood of recombination between a parent-child pair is extremely small. For example, a rough estimation of the relationship of genetic distances and physical distances is about $1 \mathrm{Mbps} / \mathrm{cM}$. The average marker interval distance of a 500K SNP chip is about 6 Kbps. Therefore, the probability of seeing a single recombination event from a parent-child pair of 170 SNP markers ( $\sim 1 \mathrm{Mbps})$ is only $\sim 1 \%$. One can assume a child inherits an entire haplotype segment from a parent for a sufficiently large number of SNPs (i.e., zero recombinant assumption). In such a case, the inheritance behavior between a parent-child pair is unique throughout all $m$ loci, and it is convenient and practically appealing to use a single binary variable $(h)$ to indicate the inheritance behavior between a parentchild pair.

Definition 2.2. Inheritance variable $h^{x_{1} x_{2}} \in\{0,1\}$ is defined between a parent $x_{1}$ and a child $x_{2}$. $h^{x_{1} x_{2}}=0$ if $x_{2}$ inherits the paternal haplotype of $x_{1}, h^{x_{1} x_{2}}=1$ if $x_{2}$ inherits the maternal haplotype of $x_{1}$.

\subsection{Mendelian constraints as a linear system}

Mendelian laws of inheritance impose constraints on $p s$ and $h$ variables for each parent-child pair at each locus. These constraints can be represented by a linear relationship of $p s$ and $h$ variables over the group $\left(Z_{2},+\right)($ where $0+0=0,0+1=1,1+1=0)$. Table 1 summarizes all cases of constraints at a certain locus $i$ for a parent-child pair. When an individual is homozygous at a certain locus, its $p s$ variable at this locus is determined by definition. When one or both of the parents of an individual are homozygous at a certain locus, this individual's $p s$ variable at this locus is also determined. In both cases, the $p s$ variable is pre-determined. In all the other cases, there is a constraint for each parent-child pair between $p s$ variables and the $h$ variable, as shown in the last three cases in Table 1. The constraints introduced by the zero recombinant assumption is enforced by the single $h$ variable between each parentchild pair. Therefore, the system formed by the sets 
of constraints collected based on Table 1 consists of all the constraints from data. The satisfiability (or consistency) of this system is equivalent to whether there is a zero recombinant solution.

Table 1. Constraints for a parent-child pair $x, y$.

\begin{tabular}{c|c|l|l|l}
\hline \multicolumn{2}{c|}{ genotype } & \multicolumn{3}{c}{ constraint } \\
\hline$x$ & $y$ & & if $x$ is father & if $x$ is mother \\
\hline $1 / 1$ & $1 / 2$ & $p_{i}^{x}=0$ & $p_{i}^{y}=0$ & $p_{i}^{y}=1$ \\
$2 / 2$ & $1 / 2$ & $p_{i}^{x}=0$ & $p_{i}^{y}=1$ & $p_{i}^{y}=0$ \\
$1 / 2$ & $1 / 2$ & & $p_{i}^{y}=p_{i}^{x}+h^{x y}$ & $p_{i}^{y}=p_{i}^{x}+h^{x y}+1$ \\
$1 / 2$ & $1 / 1$ & $p_{i}^{y}=0$ & $p_{i}^{y}=p_{i}^{x}+h^{x y}$ & $p_{i}^{y}=p_{i}^{x}+h^{x y}$ \\
$1 / 2$ & $2 / 2$ & $p_{i}^{y}=0$ & $p_{i}^{y}=p_{i}^{x}+h^{x y}+1$ & $p_{i}^{y}=p_{i}^{x}+h^{x y}+1$ \\
\hline
\end{tabular}

\subsection{Locus graphs}

To process constraints, Xiao et al. ${ }^{15}$ introduced the concept of locus graphs. We give a brief introduction here for the sake of completeness. A locus graph $L_{i}\left(V, E_{i}\right)$ is constructed for each locus $i$ to record the constraints on $h$ variables. $V$ consists of all individuals as nodes. There exists an edge in $E_{i}$ between a parent-child pair only if the $p s$ variables of this pair is constrained on the correspondent $h$ variable, i.e., when the parent is heterozygous at locus $i$ (the last three cases in Table 1). Each edge is also labeled by the $h$ variable and the constant associated with the constraint. We refer to this kind of constraints (a linear equation consists of $p s$ variables and a $h$ variable) as edge constraints. Figure 2(b) shows an example of a locus graph.

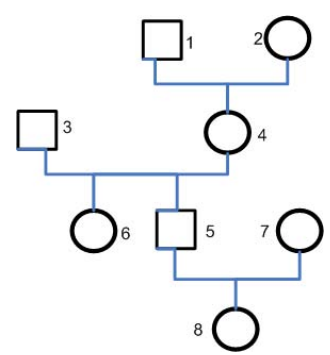

(a)

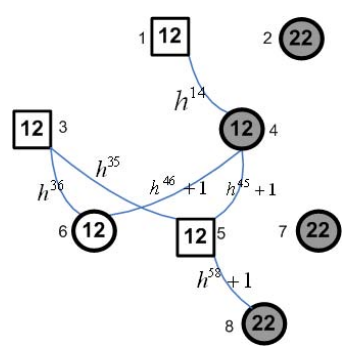

(b)
Fig. 2. (a) A pedigree with 8 members. (b) Given the genotype at a certain locus $i$, the correspondent locus graph $L_{i}$ and $h$ variable constraints. ps variables of shaded members $(2,4,7,8)$ are pre-determined. From this locus graph, we can generate two non-redundant $h$ variable constraints, one is a cycle constraint, $h^{35}+h^{36}+h^{45}+h^{46}=0$ (formed by individual $3,4,5,6)$, the other is a path constraint, $h^{45}+h^{58}=0$ (from individual 4 to 8 via 5 ).

The original idea of Ref. 15 was to integrate edge constraints to construct a new subsystem that only consists of $h$ variables. Their algorithm then solved the subsystem and used $h$ variable solutions to solve $p s$ variables. We also record edge constraints on locus graphs. However, instead of explicitly listing and solving the constraints on $h$ variables, we use disjoint-set structures to collect, encode and thus examine the consistency of these constraints, which help us achieve a better time complexity result to obtain a general solution.

\subsection{Linear constraints on $h$ variables}

There are essentially two types of constraints on $h$ variables in a locus graph $L_{i}$ : path constraints and cycle constraints. Notice that the classification of constraints here is more succinct than those in previous work ${ }^{12,15}$ because our method of handling constraints does not require further discrimination of them. According to Table 1, each edge $e_{x y}$ in a locus graph represents an edge constraint in the form $p_{i}^{x}+p_{i}^{y}=h^{x y}+c_{i}^{x y}$, where $c_{i}^{x y}$ is a constant $\in\{0,1\}$. We use a subscript $i$ for $c_{i}^{x y}$ because for different loci, the constant between a parent-child pair may be different, which depends on the genotype at that locus as specified in Table 1. For a path $P_{\widetilde{v_{s}, v_{t}}}$ from individual $s$ to individual $t$ in locus graph $L_{i}$, if we sum up all edge constraints on this path, we have

$\sum_{e_{x y} \in P_{\widehat{v_{s}, v_{t}}}}\left(p_{i}^{x}+p_{i}^{y}\right)=p_{i}^{s}+p_{i}^{t}=\sum_{e_{x y} \in P_{\widehat{v_{s}, v_{t}}}}\left(h^{x y}+c_{i}^{x y}\right)$.

If $p_{i}^{s}$ and $p_{i}^{t}$ are pre-determined constants, we end up with a path constraint on $h$ variables, which is

$$
\sum_{e_{x y} \in P_{\widetilde{v_{s}, v_{t}}}} h^{x y}=p_{i}^{s}+p_{i}^{t}+\sum_{e_{x y} \in P_{\widetilde{v_{s}, v_{t}}}} c_{i}^{x y}
$$

where the right-hand side is a constant. Similarly, for a cycle $C$ in locus graph $L_{i}$, which may exist even on a tree pedigree (e.g., when a nuclear family has more than one heterozygous children), we sum up all edge constraints on $C$,

$$
\sum_{e_{x y} \in C}\left(p_{i}^{x}+p_{i}^{y}\right)=0=\sum_{e_{x y} \in C}\left(h^{x y}+c_{i}^{x y}\right),
$$

and finally have a cycle constraint on $h$ variables

$$
\sum_{e_{x y} \in C} h^{x y}=\sum_{e_{x y} \in C} c_{i}^{x y} .
$$




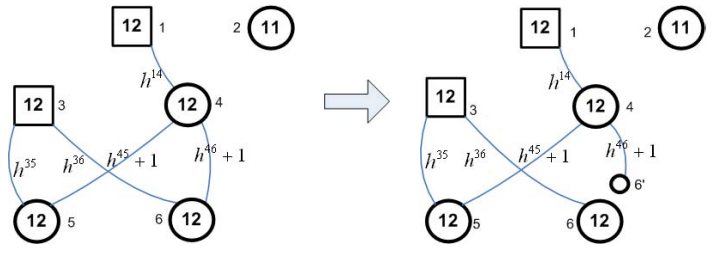

(a)

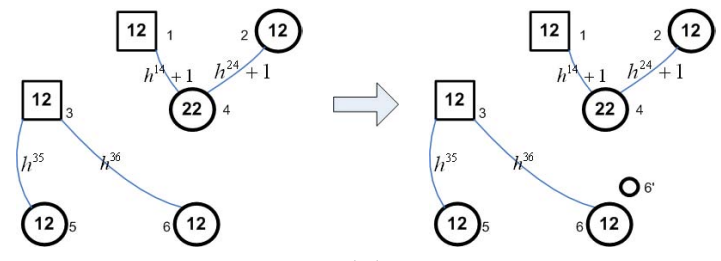

(b)

Fig. 3. Node splitting applied to a nuclear family at two loci to remove local cycles. (a) The original locus graph (left), and the locus graph (right) with edges remounted after node 6 was duplicated. (b) A locus graph at another locus before (left) and after (right) node 6 was split. Though no local cycle exists in the locus graph in b, node 6 was also duplicated so that all locus graphs will still have the same number of nodes after splitting.

\section{METHODS}

By exploiting special features of the constraints on $h$ variables, it is not necessary to explicitly list every path and cycle constraint to check their consistency. We employ disjoint-set structures to detect and to check the consistency of constraints on $h$ variables. For each locus graph $L_{i}$, we build a disjoint-set structure $D_{i}$ to encode its connectivity information. We update the disjoint-set structure incrementally upon processing each edge constraint on a locus graph. Path constraints on a locus graph are detected during this process and will be stored in another disjoint-set structure $D$. The whole algorithm works on $m+1$ such disjoint-set structures, one $D_{i}$ for each locus graph $L_{i}$ and one $D$ for encoding all path constraints. In this section, we assume the inputs are tree pedigrees with complete data. Cycles on a locus graph from a tree pedigree can only be generated within a nuclear family when it has multiple children. We first discuss a node splitting strategy in subsection 3.1 to break all such short cycles, to obtain only path constraints for further processing. Construction of $D_{i}$ from each locus graph $L_{i}$ to detect path constraints will be discussed in subsection 3.2. Processing of constraints and consistency check will be discussed in subsection 3.3 and a general solution of $h$ variables will be decoded from the disjoint-set structure $D$. Solutions of $p s$ variables will then be obtained. The analysis of time complexity and correctness of the algorithm on tree pedigrees will be discussed in subsection 3.4. One of the advantages of the proposed algorithm is that it can be easily extended to the general cases of looped pedigrees and pedigrees with missing data. And we show these extensions in section 4 .

\subsection{Split nodes to break cycles}

In order to simplify the constraint detection, we first transform cycle constraints to path constraints by breaking cycles in locus graphs. There are essentially two kinds of cycles in a locus graph: global cycles that are introduced by marriages between relatives and local cycles that are introduced by multiple children within one nuclear family (e.g., Figure $2(\mathrm{~b})$ ). Only local cycles will exist in a tree pedigree and will be dealt with in this subsection. The treatment of global cycles will be deferred to subsection 4.1 when we discuss the extension to looped pedigrees. We break local cycles for each nuclear family with multiple children by splitting some child nodes and by remounting their edges on each locus graph. More specifically, when a nuclear family has multiple children, any child node $v$ (except an arbitrarily fixed one $v_{0}$ ) and its genotypes will be duplicated to create a new node $v^{\prime}$ in the same manner across all locus graphs. New ps variables will be introduced for these duplicated nodes. For each splitting node $v$, the edge from its mother (if there is) will be reconnected to node $v^{\prime}$. All other edges regarding node $v$ remain untouched. Figure 3 shows an example on how node splitting is performed. By doing so, we technically avoid the treatment of cycle constraints. After the duplication, all new locus graphs (actually locus trees now) still have the same set of nodes. Notice that one has to record all local cycle constraints on $h$ variables and constraints that the $p s$ variables of duplicated nodes must have the same assignments as those in their original copies. Their constraints can be easily dealt with for local cycles because they only involve local structures (nuclear families). This will be further discussed in the next subsection. 


\subsection{Detect path constraints from locus graphs}

We develop an incremental procedure to detect all path constraints from a locus graph by utilizing a disjoint-set structure. As we can see from the constraints on $h$ variables in Equation 1, a path constraint is specified by the $p s$ variables of its end nodes and summation of the constant parity value $c_{i}^{x y}$ associated with the edge constraint on each of its edges. Our goal is to detect each non-redundant path on a locus graph with pre-determined end nodes and meanwhile obtain the constant parity summation associated with that path.

To do so, we maintain a disjoint-set structure $D_{i}$ for each locus graph $L_{i}$ and update it incrementally. The disjoint-set structure is defined by a pair of values $\operatorname{rep}_{i}[v]$, off $\operatorname{set}_{i}[v]$ for each node $v$ in $V\left(L_{i}\right)$. We use subscript $i$ here to emphasize that the disjoint-set structure $D_{i}$ is specific for each locus graph. $\operatorname{rep}_{i}[v]$ indicates the node which acts as the representative of the set containing $v$. And the offset of a node of $\operatorname{set}_{i}[v]$ indicates the summation of the constants associated with the edge constraints on the path from $v$ to its $r e p_{i}$. Namely, if $r e p_{i}[v]=v_{0}$, then

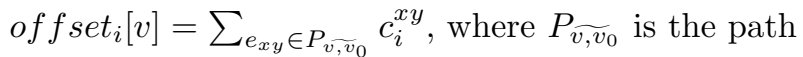
with end nodes $v$ and $v_{0}, c_{i}^{x y}$ is the constant associated with the edge constraint on edge $e_{x y}$ (as specified in the last 3 cases of Table 1 ). Initially, for every node in $V: \operatorname{rep}_{i}[v] \leftarrow v$, off set $\left.i v\right] \leftarrow 0$. We examine each $e^{x y} \in L_{i}$ and update $D_{i}$ by considering the edge constraint $p_{i}^{x}+p_{i}^{y}=h^{x y}+c_{i}^{x y}$ represented by $e^{x y}$. If both $p_{i}^{r e p_{i}[x]}$ and $p_{i}^{r e p_{i}[y]}$ are pre-determined, we report a path constraint and record it in $D$ for consistency check (see subsection 3.3). The two sets represented by rep $i[x]$ and $\operatorname{rep}_{i}[y]$ will always be merged into one because they are connected by an edge $e^{x y}$ and we always let one pre-determined representative be the representative of the new set if there is such one. At the end, any two nodes connected by a path in $L_{i}$ will be merged into one set and a set in $D_{i}$ only consists of connected nodes in $L_{i}$. By doing so, we can safely detect all path constraints on $L_{i}$. Furthermore, the constant associated with a path constraint between two nodes $v_{s}$ and $v_{t}$ in the same set can be reconstructed as

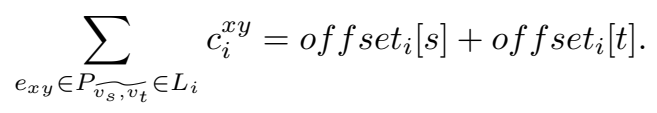

The procedure is illustrated in Algorithm 3.1.

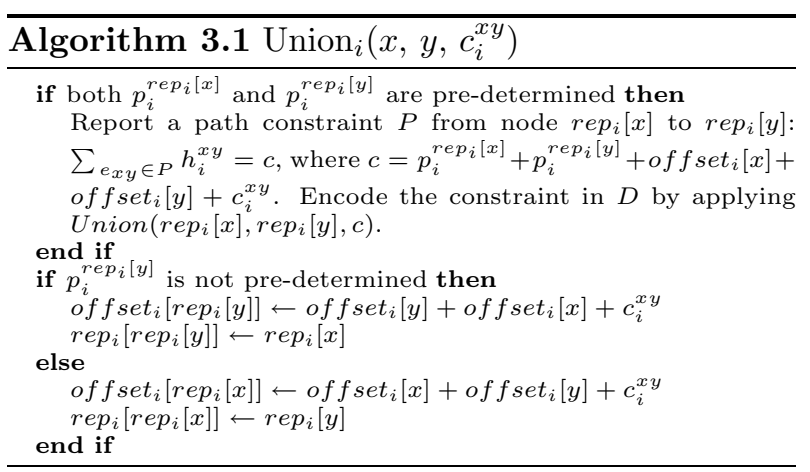

We also need to capture all constraints that may have not been processed yet in the above procedure due to node splitting. This is easy for a tree pedigree which only possibly has local cycles to split. There are three possible types of constraints that need special attentions due to node splitting, i.e., local cycle constraints themselves, $p s$ variables between duplicated nodes and their corresponding splitting nodes, and some path constraints originally existing in the locus graph before splitting, but broken by splitting. We can prove by case analysis that all these constraints can be safely recovered as path constraints on locus graphs after node splitting. We leave the proof in our extended journal version and we illustrate the cases using an example in Figure 4.

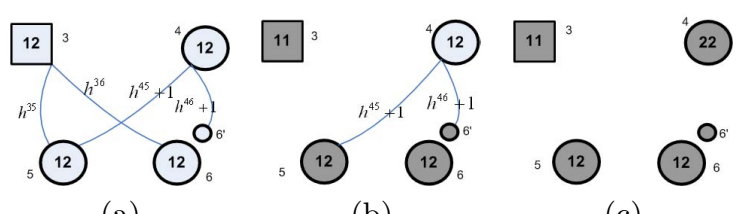

(a)

(b)

(c)

Fig. 4. This example illustrates all possible patterns of locus graphs of a nuclear family on a tree pedigree. (a) If neither of the parents is homozygous at this locus, then there should be a loop constraint, $h^{36}+h^{35}+h^{45}+h^{46}=c$. Since we split node 6 , it is expressed as a path constraint on path $P_{\widetilde{v_{6}, v_{6}}}$. Since the locus graph is still connected, no path via this nuclear family will be broken up due to the split of node 6. (b) (c) If one or both of the parents are homozygous at this locus, then both of the children are pre-determined. In this situation, path constraints such as $P_{\widetilde{v_{5}, v_{6}}}$, will only take the children as end nodes such that they remain on a consecutive path, unaffected by the split of node 6 . 
Figure 5 gives an example on how to detect constraints on a locus graph $L_{i}$. In the actual implementation of a disjoint-set forest, a node may not directly point to its set representative, we omit the details (see Ref. 4, 13) here for clear demonstration purpose.

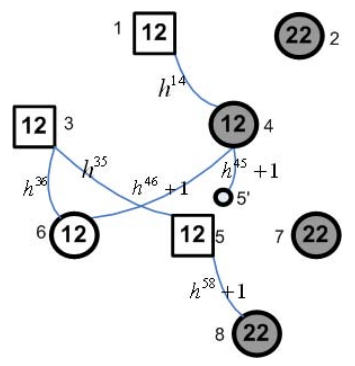

(a)

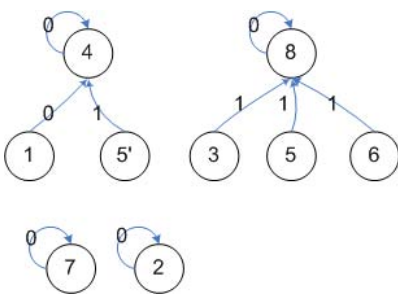

(b)
Fig. 5. An example shows the detection of all constraints from a locus graph after node splitting. (a) Locus graph $L_{i}$ of a pedigree with 8 nodes at a certain locus $i$. Shaded nodes are pre-determined. (b) The disjoint-set forest formed by adding edges 1-4, 4-5', 3-5, 3-6 and 5-8 of the locus graph $L_{i}$ in (a). No path constraint has been detected so far. We simply merge the sets containing each pair of nodes. A pointer is annotated with the offset of a node to its representative. If we further process edge 4-6 of $L_{i}$. Because both 4 and 6 have a representative with $p s$ variable pre-determined, a path between the two representatives (node 4 and 8 ) will induce a path constraint, which is $\sum_{e_{x y} \in P_{\widetilde{v_{4}, v_{8}}}} h^{x y}=0$.

\subsection{Encode path constraints in disjoint-set structure $D$}

Once we detect a path constraint, we encode this constraint also in a disjoint-set structure $D$. As usual, $D$ is defined by a pair of values $\operatorname{rep}[v]$ and of $f \operatorname{set}[v]$ for each node $v \in V$. $\operatorname{rep}[v]$ is a pointer to a node and offset $[v] \in\{0,1\}$ is a constant. We maintain this disjoint-set structure $D$ such that any two nodes $k$ and $l$ in the same set encode a path constraint in the form of $\sum_{e_{x y} \in P_{\bar{k}, l}} h^{x y}=$ offset $[k]+$ offset $[l]$. Initially, $\operatorname{rep}[v] \leftarrow v$, of f set $[v] \leftarrow 0$, for any $v \in V$. When processing a path constraint $\sum_{e_{x y} \in P_{\overline{i, j}}} h^{x y}=$ $c$, we check whether the representatives of the two end nodes $i$ and $j$ are the same. If they are not the same, which means no constraints on $h$ variables between these two nodes have been discovered so far, we merge the two sets represented by $\operatorname{rep}[i]$ and $\operatorname{rep}[j]$ as illustrated in Algorithm 3.2. When rep $[i]$ and rep $[j]$ are the same (a constraint already exists before seeing the current constraint), we must check the consistency and redundancy between the current constraint and the previous constraint. This can be easily done by comparing the constant $c$ associated with the new constraint and the constant associated with the existing constraint of $f \operatorname{set}[i]+$ of $f \operatorname{set}[j]$. If the two constants are the same, the new constraint is redundant and will be dropped; otherwise, inconsistency exists and the program reports no solutions with zero recombinations and terminates. The procedure is summarized in Algorithm 3.2.

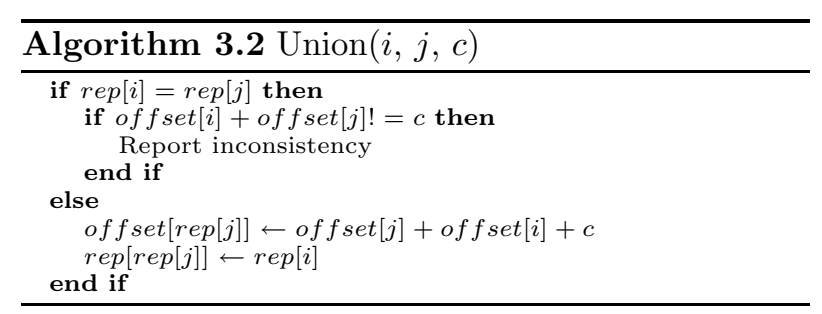

After all path constraints have been processed, the nodes will form several independent sets. A general solution of $h$ variables can be easily decoded from $D$. More specifically, for each set representative $v$ of $D$, we define a free binary variable $\alpha_{v}$ (notice $\alpha_{v}$ is not the same as $p s$ variables). A general solution of $h$ variables can be represented by a linear system of $\alpha$ variables (which are all free) in the form of

$$
h^{x y}=\alpha_{r e p[x]}+\text { offset }[x]+\alpha_{r e p[y]}+\text { off } f e t[y]
$$

We can prove that such a solution satisfies all path constraints. We can further argue that there are no other $h$ variable assignments that satisfy all path constraints. We leave the proof in our extended journal version.

Next, let's consider how to compute ps variable solutions from $h$ variable solutions. For each node $v$ in $D_{i}, v$ is connected to its set representative $\operatorname{rep}_{i}[v]$ through a path $P$ on $L_{i}$. We have $p_{i}^{v}+$ $p_{i}^{r e p_{i}[v]}=\sum_{e_{x y} \in P \in L_{i}}\left(h^{x y}+c_{i}^{x y}\right)=\sum_{e_{x y} \in P} h^{x y}+$

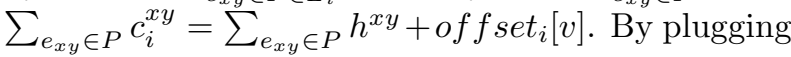
in the solution of $h$ variables in Equation 2, we will finally get a general solution for the ZRHC problem,

$$
\begin{aligned}
& p_{i}^{v}=p_{i}^{r e p_{i}[v]}+\alpha_{\text {rep }\left[\operatorname{rep}_{i}[v]\right]}+\operatorname{offset}\left[\operatorname{rep}_{i}[v]\right]+
\end{aligned}
$$

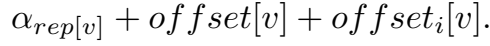

If $p_{i}^{r e p_{i}[v]}$ is not pre-determined, we have one more degree of freedom in Equation 3. 


\subsection{Analysis of the algorithm on tree pedigrees with complete data}

The overall algorithm is summarized in Algorithm 3.3. We omit the preprocessing steps (such as node splitting, construction of locus graphs) because all those operations can be done in linear time. Here we also state our main result of the algorithm as a theorem. We leave the proof in the extended journal version due to space limitation.

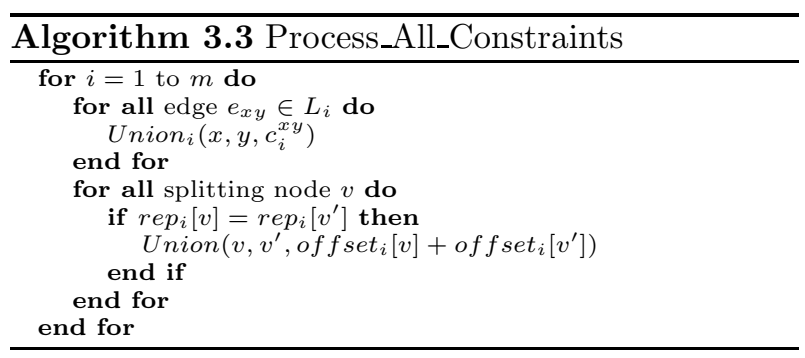

Theorem 3.1. For a tree pedigree with complete data, Algorithm 3.3 correctly outputs a general solution (Equation 2 and 3) and the number of specific solutions (degrees of freedom) for the ZRHC problem if it has a solution, and reports inconsistency otherwise. Its running time is bounded from above by $O(m n \alpha(n))$, where $m$ is the number of loci, $n$ is the number of individual and $\alpha()$ is the inverse Ackermann function ${ }^{4}$.

\section{EXTENSION TO GENERAL CASES}

\subsection{Pedigrees with mating loops}

We can further extend the above algorithm to pedigrees with mating loops and pedigrees with missing data. For a looped pedigree, we apply a similar splitting rule to locus graphs as we did for a tree pedigree, except that for a mating between two relatives all their children are duplicated in order to break a global cycle. We use the same method described in section 3.2 and 3.3 to detect all path constraints on each locus graph. However, Theorem 3.1 does not hold anymore in this case because the method does not guarantee the detection of all necessary constraints. The difference lies in the detection of path constraints broken by splitting nodes. All such path constraints can be recovered when breaking a local cycle but may not be recovered when breaking a global cycle. Figure 6 gives such an example on a looped pedigree.

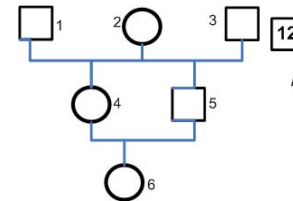

(a)

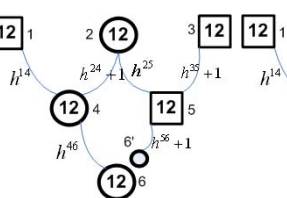

(b)

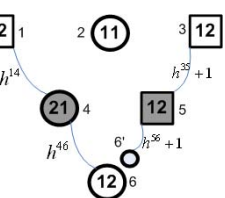

(c)
Fig. 6. An example of constraints on a looped pedigree. (a) A pedigree with a mating loop, where node 6 is produced by the mating of two relatives 4 and 5. (b) One locus graph $L_{i}$, where there is a path constraint $\sum_{e_{x y} \in P_{\widetilde{v_{6}, v_{6}}}} h^{x y}=$ $h^{24}+h^{25}+h^{46}+h^{56}=0$. (c) Another locus graph $L_{j}$, where there is a constraint $h^{46}+h^{56}=0$. Due to the splitting at node 6 , this constraint is not on a consecutive path.

Although the set of constraints are not sufficient, we can still obtain all the solutions for a looped pedigree using the following procedure. If there are already inconsistent constraints during consistency check, no solutions with zero recombinant exist. Otherwise, all the $h$ variable solutions obtained based on the general solution (Equation 2) will be examined. If a specific $h$ variable assignment is not consistent with the genotype, we simply drop that assignment. Otherwise, it will result in real haplotype solutions. To check the consistency of an $h$ variable assignment with existing genotypes, we use another disjoint-set structure to encode constraints on alleles. This step is the same for pedigrees with loops and pedigrees with missing data. Essentially for looped pedigrees, we avoid cycle constraints by splitting nodes with the expense that we may miss some constraints. We start to enumerate $h$ variables after processing existing partial constraints. However, as it will be shown in the experiment, the number of all possible $h$ variable assignments from this set of partial constraints is usually very small for a pedigree with complete data, and in most times there is only one solution for pedigrees with 20 or more loci. Therefore, the above extension can efficiently handle looped pedigrees in practice.

\subsection{Pedigrees with missing data}

For an algorithm to be practically useful, it has to be applicable on real data. Most real data contains missing. One advantage of the proposed algorithm is that it can be easily extended to deal with missing data. Extension of existing work ${ }^{3,12,15}$ to handle missing is not trivial at all. We take a similar approach as in subsection 4.1 to deal with missing 
data. Partial constraints on $h$ variables will be collected based on existing genotype data. Solutions of $h$ variables will be obtained based on the set of partial constraints and will be checked for consistency with existing genotype data. More specifically, for a pedigree with missing data, we construct the locus graph $L_{i}$ for each locus $i$ as usual with node splitting if necessary. The edges in $L_{i}$ will only be constructed by examining every parent-child pair whose genotypes are complete at this locus $i$. We apply Algorithm 3.3 to process all edge constraints from such locus graphs. And from the partial constraints on $h$ variables, we get a solution in its general form (Equation 2). The degree of freedom, which is $n_{D}-1$ where $n_{D}$ is the number of independent sets, usually is significantly smaller than the degree of freedom of the original $h$ variables without constraints, which is usually close to $2 n$. Therefore, our algorithm has the potential to be significantly faster than those algorithms based on the enumeration of all possible $h$

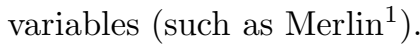

For each specific $h$ variable assignment, the compatibility check with the input genotype data is also examined by utilizing another disjoint-set structure on allele variables. For space limitations, we leave the details in the extended journal version. By doing so, we can efficiently check the consistency between a given $h$ variable assignment and the input genotype data, and generate a set of assignments of alleles that are consistent with the $h$ variable assignment. The total number of $h$ variable assignments is $2^{n_{D}-1}$, and for each assignment, the complexity of genotype consistency check is $O(m n \cdot \alpha(n))$.

\section{EXPERIMENTAL RESULTS}

We study the performance of our program (denoted as DSS) under different settings (pedigree size, number of loci, missing rate, pattern of missing) and compare its performance with two representative programs Merlin ${ }^{1}$ and PedPhase (the integer linear programming ILP algorithm in Ref. 9). Merlin is one of the most widely used statistical packages for linkage analysis and we only use its haplotyping functionality in this comparison. It also uses the zero recombinant assumption. But it examines all possible configurations of inheritance variables and only outputs those compatible ones. PedPhase.ILP is an- other widely used rule-based algorithm developed by our own group. It can produce all optimal haplotype solutions with minimal recombinants for any pedigree structures with missing data. It can solve the zero recombinant problem as a special case. But because it does not use the zero recombinant assumption explicitly, its efficiency is expected to be inferior to the current algorithm. Under the zero recombinant assumption, all three methods are exact algorithms that output all compatible solutions. Our experiments show that their implementations indeed generate the same set of haplotype assignments on same inputs. This again shows that the ZRHC formulation is valid for tightly linked markers, and the set of solutions is the same as the set of solution obtained based on likelihood approaches. Therefore, we only present results on the efficiency comparison.

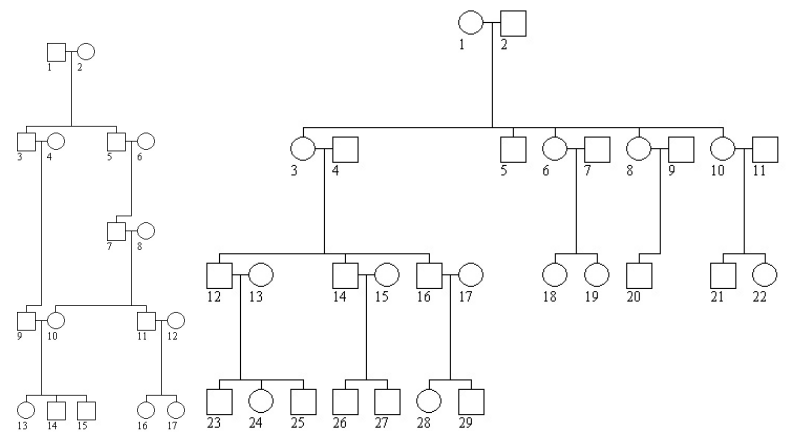

(a)

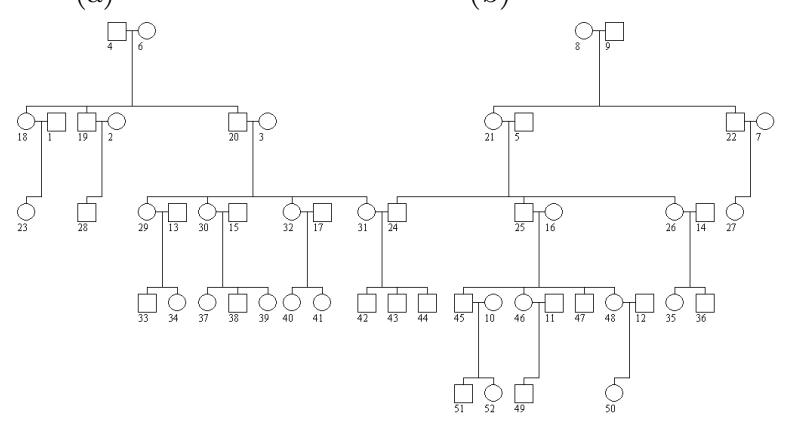

(c)
Fig. 7. Pedigree structure used in simulation.

We test all three approaches on different sizes of pedigrees $(17,29,52,128)$, all are real human pedigree structures obtained from literatures. Different number of loci $(20,50,100,200)$, different missing rates $(0.05,0.10,0.15,0.20)$ and different missing patterns are considered. We run Merlin and DSS on a Linux machine with two 3.0GHz Quad-Core Xeon 


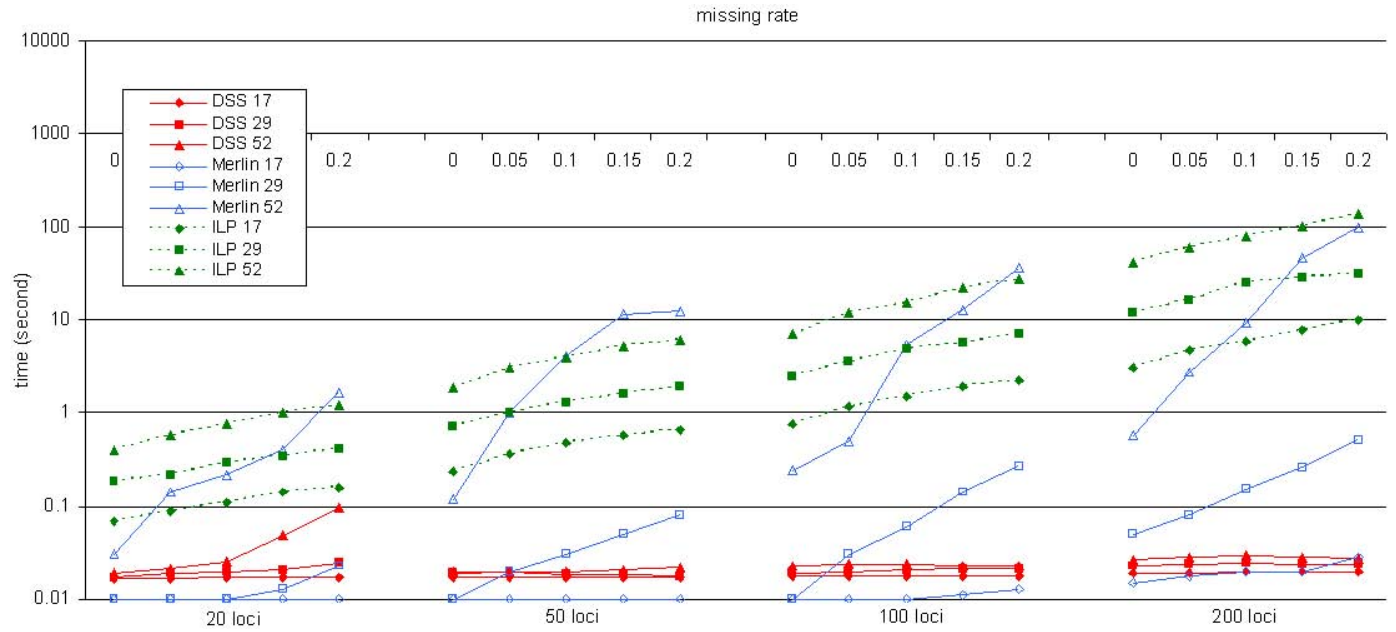

(a)

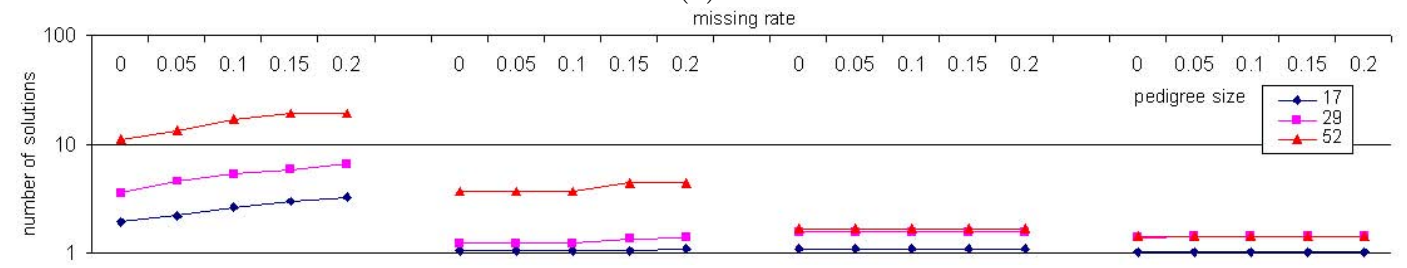

(b)

Fig. 8. (a) Comparison of running time (in seconds). (b) Average number of solutions.

5365 processors and $16 \mathrm{G}$ memory. PedPhase.ILP only has a Windows version, and it was tested on a much slower Windows machine with a much small memory (Pentium $43.2 \mathrm{GHz}$ with $2 \mathrm{G}$ memory). We measure the time needed for each of the algorithms to output all possible haplotyping solutions of a pedigree. Due to hardware limitations, the result of PedPhase.ILP on pedigree size 128 is not acquired. To generate genotype data that closely resemble real data, we use the Simulated Rheumatoid Arthritis (RA) Data from Genetic Analysis Workshop (GAW) 15. Chromosome 6 of GAW data mimics a $300 \mathrm{~K}$ SNP chip with an average inter-marker spacing of 9,586 bp. The beginning 20, 50, 100 and 200 loci are truncated to test the three algorithms. Population haplotype frequencies are calculated based on the true haplotype assignments in the simulated data, and are then fed to $\mathrm{SimPed}^{7}$, together with each pedigree structure. SimPed will then sample founder haplotypes based on their population frequencies and generate genotype data for each member in a pedigree assuming no recombinations. The three pedigree structures are shown in Figure 7, among which the pedigree with size 17 (Figure $7(\mathrm{a})$ ) is a looped one. The pedigree with size 128 is too large to fit in one page and will be provided on our website.

We designate two ways to generate samples with missing data so as to examine the behavior of the methods with respect to both missing rate and missing pattern variations. We generate the first set of samples by randomly assigning a locus to be missing at a specified missing rate. Second, we make all top generation of a pedigree completely missing for all loci, which is common in real data. For each testing category, we simulate 100 independent data sets and report the average running time. For the random missing case, Figure 8(a) shows the running time of the three programs under different settings, except for the pedigree size 128, for which the running time of Merlin is too large to be juxtaposed with DSS. The result on the pedigree with size 128 is listed in Table 2. The running time of Merlin increases exponentially with the pedigree size, the number of loci and also the missing rate. The running time of PedPhase.ILP (on a slower machine) also has an exponential growth with the increase of the missing rate 
Table 2. Comparison of running time (in seconds) between DSS and Merlin on pedigree size 128 . The running time of Merlin under some data settings exceeds an hour, and are thus omitted from our measurement.

\begin{tabular}{c|c|c|c|c|c|c|c|c|c|c}
\hline number of loci & \multicolumn{9}{|c|}{20} & \multicolumn{5}{c}{50} \\
\hline missing rate & 0.00 & 0.05 & 0.10 & 0.15 & 0.20 & 0.00 & 0.05 & 0.10 & 0.15 & 0.20 \\
\hline DSS & 0.0267 & 0.1539 & 0.3517 & 0.4991 & 0.6540 & 0.0259 & 0.0361 & 0.0368 & 0.0378 & 0.0360 \\
\hline Merlin & 70 & 300 & 600 & 800 & 1100 & 360 & 800 & 1000 & $>1300$ & - \\
\hline number of loci & \multicolumn{9}{|c|}{100} & \multicolumn{7}{c}{200} & \\
\hline missing rate & 0.00 & 0.05 & 0.10 & 0.15 & 0.20 & 0.00 & 0.05 & 0.10 & 0.15 & 0.20 \\
\hline DSS & 0.0311 & 0.0426 & 0.0373 & 0.0431 & 0.0461 & 0.0433 & 0.0587 & 0.0518 & 0.0575 & 0.0503 \\
\hline Merlin & 800 & 1200 & $>2400$ & - & - & - & - & - & - & - \\
\hline
\end{tabular}

and the number of loci but with a smaller constant compared to Merlin. It also shows a much smaller growth rate with the pedigree size. In contrast, DSS scales smoothly with all parameters (except for the missing rate when the number of loci is 20), and the improvement over Merlin or PedPhase.ILP is from 10 to $10^{5}$ folds for large pedigrees with large number of loci or high rate of missing. In fact, neither Merlin nor PedPhase.ILP can successfully infer haplotypes from the pedigree with size 128 when the number of marker is 200. However, DSS can obtain all solutions within 0.05 second, even for data with $20 \%$ missing. This shows that by solving the linear system based on partial constraints from existing data, we significantly reduce the enumeration space of inheritance variables. The experimental results show that when the number of loci is large, the program can still maintain the same linear complexity even for data with $20 \%$ missing. But for small number of loci, the running time of DSS increases as missing rate increases (though DSS can finish all the cases within 0.1 second). This is because the number of constraints on $h$ variables is roughly in proportion to the number of loci. So for small number of loci, the remaining degrees of freedom on inheritance variables after solving the linear system could still be high. This number could be partly reflected by the number of all compatible solutions in the end. Figure 8(b) compares the number of $h$ variable solutions in different circumstances. It grows with both the pedigree size and the missing rate, but decreases with the number of loci.

Next, we investigate the performance of all three algorithms on special missing patterns. Figure 9 gives some representative result on the pedigree with size 52, for which all individuals at the top generation (members 4, 6, 8, 9) are missing. For this pedigree, such missing equals a missing rate of $\sim 7.7 \%$. In terms of absolute time, DSS $(0.2 \sim 0.8 \mathrm{sec})$ is much better than the other two algorithms $(0.2 \sim 100 \mathrm{sec})$. However, the running time is higher than its own running time with a missing rate $10 \%$. The running time of Merlin and PedPhase.ILP on this special data set is between those of missing rate $5 \%$ and $10 \%$. DSS is somewhat sensitive to this special missing pattern because when all genotypes of an individual are missing, none of the inheritance variables between her and her parents or children could be determined. A further investigation on this special missing pattern is warranted.

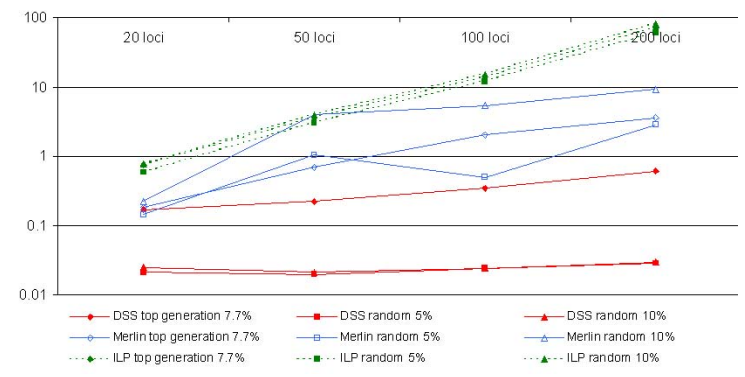

Fig. 9. Comparison of DSS and Merlin on different patterns of missing data.

\section{DISCUSSION}

We propose an algorithm for haplotype inference from pedigree data without recombinant using disjoint-set structures. The proposed algorithm can output a general solution for a tree pedigree with complete data in time $O(m n \alpha(n))$, which is a further improvement upon existing results. For a general pedigree, or a pedigree with missing data, by using the same framework, our method can significantly reduce degrees of freedom on inheritance variables and thus narrow down the search scope. Experimental results show that the algorithm is efficient in practice for both complete data and missing data, and outperforms two popular algorithms on large data 
sets. For data with large number of markers, the performance of the algorithm hardly deteriorates as the missing rate increases.

Though several theoretical results of ZRHC were recently reported ${ }^{3,12,15}$, none of them have been implemented. The empirical examination of the performance of our algorithm offers some evidence for the theoretical bounds on the complexity of such haplotyping approaches based on linear systems.

The performance of our algorithm on pedigrees with missing data depends on the number of constraints the linear system can capture. We observe that the efficiency of this linear system is influenced by variation in missing patterns. So as a possible piece of future work, we can consider a special strategy to handle individuals with all loci missing. Other possible directions are to combine the proposed algorithm with statistical approach to assign a probability likelihood for each of the assignments, and to design algorithms for whole chromosome by calling the current algorithm as a subroutine. Theoretically, it also remains open whether the linear time complexity can be observed for a general pedigree with complete data.

\section{Acknowledgement}

This research is supported by National Institutes of Health/National Library of Medicine grant LM008991, and in part by National Institutes of Health/National Center for Research Resources grant RR03655. Support for generation of the GAW15 simulated data was provided from NIH grants 5RO1-HL049609-14, 1R01-AG021917-01A1, the University of Minnesota, and the Minnesota Supercomputing Institute. We would also like to acknowledge GAW grant R01 GM031575.

\section{References}

1. Abecasis GR, Wigginton JE. Handling markermarker linkage disequilibrium pedigree analysis with clustered markers. Am. J. Hum. Genet 2005; 77: 754-767.

2. Bonizzoni P, Vedova GD, Dondi R, Li J. The haplotyping problem: an overview of computational models and solutions. J Comp Sci Tech 2003; 18(6): 675-88.

3. Chan MY, Chan W, Chin F, Fung S, Kao M. LinearTime Haplotype Inference on Pedigrees without Recombinations. Proc. of the 6th Annual Workshop on
Algorithms in Bioinformatics (WABI'06) 2006: 5667.

4. Cormen TH, Leiserson CE, Rivest RL, Stein C. Introduction to Algorithms. 2nd edition, McGraw-Hill Book Company, Boston, MA. 2003: 498-517.

5. Gusfield D. An overview of combinatorial methods for haplotype inference. Lecture Notes in Computer Science (2983): Computational Methods for SNPs and Haplotype Inference. 2004: 9-25.

6. Halldórsson BV, Bafna V, Edwards N, Lippert R, Yooseph S, Istrail S. A survey of computational methods for determining haplotypes. Lecture Notes in Computer Science (2983): Computational Methods for SNPs and Haplotype Inference. 2004: 26-47.

7. Leal SM, Yan K, Müller-Myhsok B. SimPed: a simulation program to generate haplotype and genotype data for pedigree structures. Hum Hered 2005; 60: 119-122.

8. Li J, Jiang T. Efficient Inference of Haplotypes from Genotype on a Pedigree. Journal of Bioinformatics and Computational Biology $(J B C B) 2003 ; 1(1)$ : 4169.

9. Li J, Jiang T. Computing the Minimum Recombinant Haplotype Configuration from Incomplete Genotype Data on a Pedigree by Integer Linear Programming. Journal of Computational Biology 2005; 12: $719-739$.

10. Li J, Jiang T. A survey on haplotyping algorithms for tightly linked markers. Journal of Bioinformatics and Computational Biology 2008; 6(1): 241-259.

11. Liu L, Chen X, Xiao J, Jiang T. Complexity and approximation of the minimum recombination haplotype configuration problem. In Proc. 16th International Symposium on Algorithms and Computation (ISAAC'05) 2005: 370-379.

12. Liu L, Jiang T. Linear-Time Reconstruction of ZeroRecombinant Mendelian Inheritance on Pedigrees without Mating Loops. Proc. of Genome Informatics Workshop (GIW'2007) 2007: 95-106.

13. Tarjan RE, Leeuwen J. Worst-case analysis of set union algorithms. Journal of the ACM 1984; 31(2): 245-281.

14. The international HapMap Consortium. A second generation human haplotype map of over 3.1 million SNPs. Nature 2007; 449: 851-61.

15. Xiao J, Liu L, Xia L, Jiang T. Fast Elimination of Redundant Linear Equations and Reconstruction of Recombination-Free mendelian Inheritance on a Pedigree. Proc. of 18th Annual ACM-SIAM Symoposium on Discrete Algorithms (SODA'07) 2007: 655664.

16. Zhang K, Zhao H. A comparison of several methods for haplotype frequency estimation and haplotype reconstruction for tightly linked markers from general pedigrees. Genetic Epidemiology 2006; 30(5): 423437.

17. Zhang XS, Wang RS, Wu LY, Chen L. Models and Algorithms for Haplotyping Problem. Current Bioinformatics 2006; 1(1): 105-114. 